Cite this: Phys. Chem. Chem. Phys., 2011, 13, 2663-2666

\title{
Ion conducting particle networks in liquids: modeling of network percolation and stability
}

\author{
Anna Jarosik, ${ }^{a}$ Uwe Traub, ${ }^{a}$ Joachim Maier ${ }^{* a}$ and Armin Bunde ${ }^{b}$ \\ Received 20th September 2010, Accepted 25th November 2010 \\ DOI: $10.1039 / \mathrm{c0cp01870h}$
}

Networks of inorganic particles (here $\mathrm{SiO}_{2}$ ) formed within organic liquids play an important role in science. Recently they have been considered as 'soggy sand' electrolytes for Li-based batteries with a fascinating combination of mechanical and electrical properties. In this communication we model formation and stability of the networks by Cluster-Cluster Aggregation followed by coarsening on a different time scale. The comparison of computer simulations based on our model with experimental results obtained for $\mathrm{LiClO}_{4}$ containing polyethylene glycol reveals (i) that the percolation threshold for interfacial conductivity is very small, (ii) that the networks once formed coarsen with a time constant that is roughly independent of volume fraction and size - to a denser aggregate which then stays stable under operating condition. (iii) Trapping of the conducting solvent at high packing is also revealed.

Coherent networks of solids in liquids play an important role in colloid chemistry and physics and have a broad range of technological applications. Perhaps the most popular examples are dispersion paints that consist of inorganic particles dispersed in appropriate liquids. When a shear stress is applied, the network of the particles breaks up, and as a result the overall viscosity decreases. This thixotropy is beneficial for the process of painting. Brushing exerts shear stress and the resulting thinning facilitates applying the paint. Other examples are gels in which-unlike sols - colloidal particles form percolating networks.

Here we will focus on the recently discovered "soggy sand" electrolytes in which by admixing fine insulating particles to salt containing liquids the overall ionic conductivity is pronouncedly increased. ${ }^{1,2}$ Since this effect can be attributed to preferential ion diffusion along the network of the insulating particles, these electrolytes rely particularly sensitively on the formation and stability of the network. ${ }^{3,4}$ Technologically, they are especially interesting for Li-based batteries, as they improve the mechanical properties substantially and may even exhibit higher $\mathrm{Li}^{+}$conductivities. Even more importantly, the $\mathrm{Li}^{+}$transport number can be significantly increased as the

${ }^{a}$ Max-Planck-Institut für Festkörperforschung, D-70569 Stuttgart, Germany.E-mail: s.weiglein@fkf.mpg.de; Fax: 49711 6891722; Tel: 497116891721

${ }^{b}$ Justus-Liebig Universität Gießen, Institut für Theoretische Physik III, D-35392 Gießen, Germany counter ion is immobilized. In addition, safety aspects are affected in a beneficial way. The conductivity-volume fraction characteristics are similar to the ones obtained for solid-solid composites that showed conductivity enhancement effects, but suffer from a lack of morphological stability and reproducibility.

In this Communication we refer to conductivity measurements of polyethylene-glycol containing $\left(\mathrm{LiClO}_{4}\right)$ dispersed with insulating $\mathrm{SiO}_{2}$ particles $\left(\mathrm{PEG}\left(\mathrm{LiClO}_{4}\right): \mathrm{SiO}_{2}\right)$, which from the experimental point of view will be published in greater detail elsewhere. ${ }^{5,6}$ To be precise, these oligomeric molecules are partly or fully $\mathrm{CH}_{3}$-terminated. We used PEG-150 (i.e. poly(ethylene glycol) methyl ether with a polymerization degree between 2 and 3) and PEG-350 (i.e. poly(ethylene glycol) dimethyl ether with a mean polymerization degree slightly above 7). We used two different types of $\mathrm{SiO}_{2}$ that differed only slightly in grain size but to a greater degree in the original density of surface $\mathrm{OH}$-groups, possibly leading to different network morphologies. ${ }^{7}$ In the following we denote them by $\mathrm{SiO}_{2} \mathrm{I}$ ( $7 \mathrm{~nm}$, fumed) and $\mathrm{SiO}_{2}$ II (10 nm, not fumed). Unlike previous measurements, we were able to de-convolute transient and steady state effects. This allowed us to study experimentally and understand better (a) the onset of the conductivity increase at very low volume fraction $\varphi$ of the insulating particles, (b) the decrease of the conductivity with increasing $\varphi$ after having reached a more or less sharp maximum at low $\varphi$, (c) the conductivity at large $\varphi$ that may have fallen to values smaller than at $\varphi=0$ even if corrected for the insulating portion, and finally (d) the scatter obtained from preparation to preparation, in particular at low volume fractions $\varphi$ of the insulating $\mathrm{SiO}_{2}$ particles. We are particularly interested in the low stability of the system at low packing which leads to perceptible time changes before these stationary conductivities have been reached. We show by extensive computer simulations that the key to understanding these intriguing issues is the formation of the oxide particle network and its initial coarsening. This coarsening known to be driven by the decrease in the surface free energy becomes soon kinetically frozen as we are going to show.

Fig. 1 shows as a function of $\mathrm{SiO}_{2}$ volume fraction the stationary conductivity of PEG $\left(\mathrm{LiClO}_{4}\right): \mathrm{SiO}_{2}$. While the absolute conductivity values of PEG-150 and PEG-350 are very different (according to their different molecular weights and hence viscosities), the conductivity enhancement is of the 


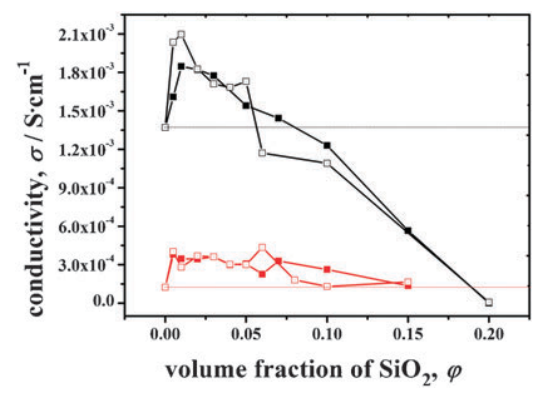

Fig. 1 Stationary ionic conductivity of $\mathrm{PEG}\left(\mathrm{LiClO}_{4}\right): \mathrm{SiO}_{2}$ as a function of the $\mathrm{SiO}_{2}$ volume fraction at room temperature, for $\mathrm{SiO}_{2}$ I (open symbols) and $\mathrm{SiO}_{2} \mathrm{II}$ (full symbols). The upper two curves are for PEG-150, while the two lower curves are for PEG-350. Note that for PEG-150 the conductivity curves at large $\varphi$ values have been fallen below their $\varphi=0$ value.

same order of magnitude in both cases. The results on PEG-350 are consistent with results obtained in ref. 8 and 9 . (The mobility effect roughly cancels in the ratio $\sigma(\varphi) / \sigma(0){ }^{5,10}$ )

Fig. 2 displays the time dependence of the ionic conductivity. ${ }^{11}$ Fig. 2a shows that a denser packing of the $\mathrm{SiO}_{2}$ particles leads to a smaller conductivity decrease with time, while the relaxation time $\tau$ stays more or less constant in all these cases. This insensitivity of the relaxation time is very typical and is also valid for variation of the chain length of PEG, for the different types of $\mathrm{SiO}_{2}$ as well as for different salt concentrations. It is even the case if we compare different degrees of polymerization. However, only if we vary grain size drastically (from $10 \mathrm{~nm}$ to $1 \mu \mathrm{m}$ ) a significant increase of $\tau$ is observed. ${ }^{6}$ These phenomena find their explanations in the coarsening kinetics. Here we are not referring to the local mechanism of conductivity enhancement, ${ }^{5}$ which we believe is the Heterogeneous Doping mechanism, ${ }^{12}$ i.e. anion adsorption at the $\mathrm{SiO}_{2}$ surfaces leading to breaking up ion pairs, thus increasing the concentration of free $\mathrm{Li}$ ions and hence the $\mathrm{Li}^{+}$ surface conductivity. Here we are interested in how these local interfacial (space charge) effects percolate along the $\mathrm{SiO}_{2}$

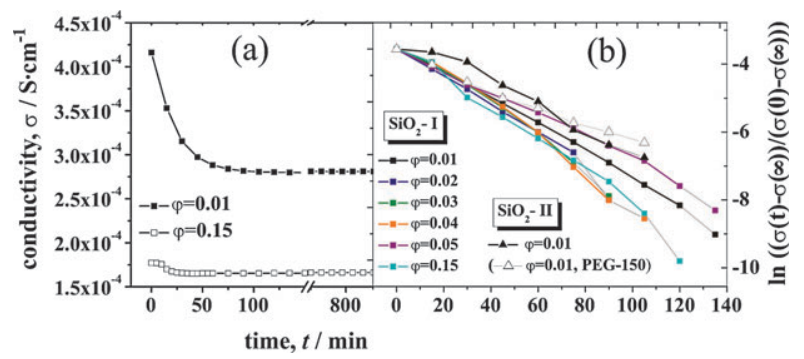

Fig. 2 Transients of the ionic conductivity of the soggy-sand electrolytes after preparation. Fig. 2a shows the conductivity of PEG-350 $\left(\mathrm{LiClO}_{4}\right): \mathrm{SiO}_{2}$ for fixed grain size $\left(\mathrm{SiO}_{2} \mathrm{I}\right)$ and two volume fractions $\varphi=0.01$ and $\varphi=0.15$ of the insulating $\mathrm{SiO}_{2}$ particles. While the absolute values of both conductivities are very different, their relaxation times $\tau$ are quite similar. This is better seen in Fig. 2b where in a semi-logarithmic plot, the normalized decay of the conductivity for several values of $\varphi$ between 0.01 and 0.15 for PEG-350 containing $\mathrm{SiO}_{2} \mathrm{I}$ is shown. For a first-order kinetics one expects a straight line with a slope characterizing $\tau$. For comparison, we also show results for the smallest volume fraction $\varphi=0.01$ for $\mathrm{SiO}_{2}$ II in PEG-350 and PEG-150. networks formed, which we believe is the key to understanding the intriguing features of the soggy sand electrolytes.

For clarity, Fig. 3 summarizes the main features of the conductivity of the soggy sand electrolytes in a sketch.

Before we start with the description of the modeling let us consider a few very simple details: If all the particles were randomly distributed in a $d$-dimensional cube of length $L$ ( $L$ : distance between electrodes), as it is in the very beginning of colloidal suspension, the mean distance between particles of diameter $R$ scales as $\bar{\delta} \propto R / \varphi^{1 / 3}$. This weak dependence $\varphi$ compared with the dependence on $R$ is the reason why nanosized silica leads to a significant particle density even at very low volume fractions. On the other hand, even in close-packed structures of dense spherical particles, there is still $26 \%$ free volume that is percolating and available for the electrolyte. It is also illustrative to artificially assume that all the solid particles form parallel chains of diameter $R$ and length $L$ : then these chains have a mean distance that scales as $\bar{\delta} \propto R / \varphi^{1 / 2}$ and the mean number of chains $\bar{N}$ per (electrode) area scales as $\bar{N} \propto \varphi / R^{2}$. It is obvious that a proportional decrease of particle diameter increases the mean crowdedness more than a proportional increase of volume fraction does.

The realistic network formation occurs by formation of fractal networks, as $\mathrm{SiO}_{2}$ particles randomly overcome the colloidal repulsion threshold and then are irreversibly bound by stronger van-der-Waals or even covalent interactions. ${ }^{13-15}$ To estimate the percolation threshold $\left(\varphi_{c}\right)$ of such networks, we note that at $\varphi_{c}$ the fractal network starts spanning the space, which in our case is a d-dimensional cube of length $L$. By definition, the volume concentration $\varphi$ of $N$ particles of diameter $R$ in this cube is $\varphi \propto N(R / L)^{d}$. At $\varphi_{c}, N$ scales with $L$ as $N \sim(L / R)^{d_{f}}$ where $d_{f}$ is the fractal dimension of the network. This yields $\varphi_{c} \propto(L / R)^{d_{f}}(R / L)^{d}=(L / R)^{-\left(d-d_{f}\right)}$, which tends to zero in the limit of $L / R \rightarrow \infty$.

We model our experiments in two steps (see Fig. 4). First we let the particles form a network by an irreversible diffusioncontrolled cluster-cluster aggregation process (transition from left hand side column to center column). In Fig. 4, the sticking probability is one, but we also allowed for lower $p$-values. Then on a different time scale (this deconvolution is justified by the experiments) we let them coarsen. (Transition from center column in Fig. 4a to right hand side column.)

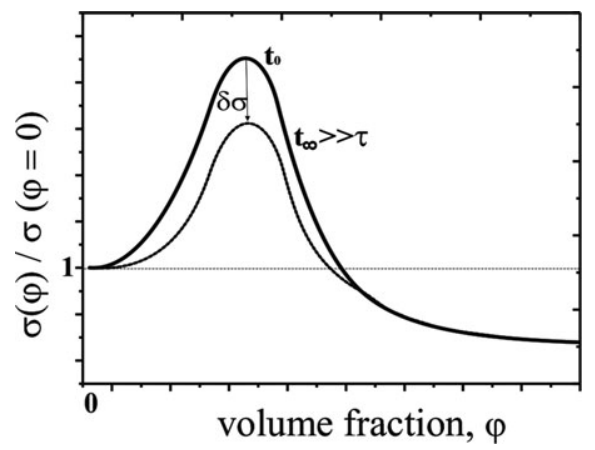

Fig. 3 Schematic plot of ionic conductivity versus $\mathrm{SiO}_{2}$ volume fraction for freshly prepared material $\left(\sim t_{0}\right)$ and for the stationary state $\left(t_{\infty}\right)$. Note that the $\mathrm{SiO}_{2}$ network formation happens fast between $t=0$ and $t=t_{0}$. Position and height of the maximum is strongly parameter dependent. 


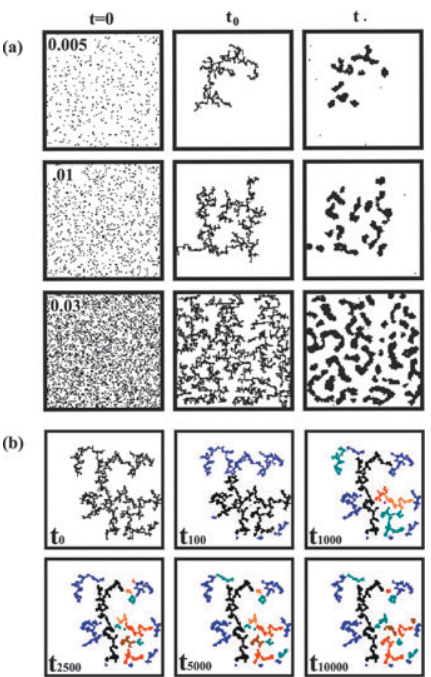

Fig. 4 (a) Fractal networks formed from the starting configuration (left column, $t=0$ ) by cluster-cluster aggregation (center column, $\left.t=t_{0}\right)$. The particle concentrations are $0.005,0.01$, and 0.03 , respectively. The networks are then allowed to coarsen (right column, final situation of the coarsening process $t=t_{\infty}$ ). The sticking probability is $100 \%$. (b) The two bottom rows show that the coarsening slows down very quickly already at 1000 Monte-Carlo steps when essentially the steady state $t_{\infty}$ is reached (colours highlight disconnected clusters).

For simplicity, we consider a two-dimensional (2D) system, since we do not expect to obtain greater insight from 3D-simulations. On the same grounds, it also suffices to consider network morphology rather than calculating 2D conductivities. Since the cluster-cluster aggregation has been described extensively in the literature (see, e.g. ref. 16), we only describe here the coarsening process which we believe captures the essential physics despite of the simplicity of the approach.

In the coarsening process (see Fig. 5) the fractal aggregate tends to minimize its surface energy. To model this process by a Monte-Carlo simulation, we first choose randomly an aggregate particle and count its nearest and next nearest neighbors. We assume that one nearest neighbor contacted via full edge contact provides the interaction energy $\Delta$ and contact via corner connection (or half corner connection, see below) provides $\Delta / 2 .{ }^{17}$ For an illustration, see Fig. 5, where the initial state energy for the red particle is $E_{I}=2 \Delta+2 \Delta / 2=3 \Delta$. In the next step, we analyze the number of possible movements

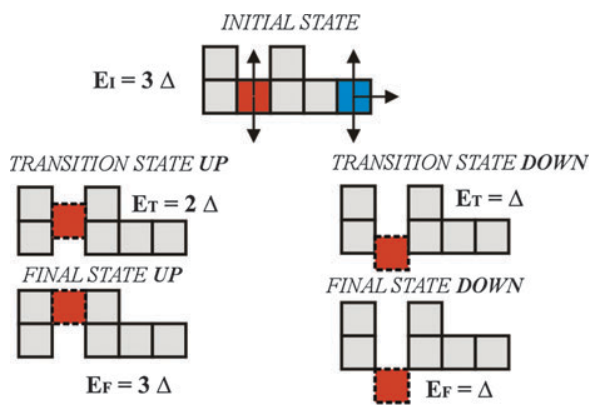

Fig. 5 Typical kinetic steps in the simulation of the coarsening process, for a partice with a two-side contact (red square) and a particle for a one-side contact (blue square). of the chosen particle which prefers to change its state to an energetically more favorable one and in practice result in a more compact cluster. In the example of Fig. 5, apart from staying, the chosen particle can move 'up' or 'down'. The process as considered is not precisely leading through a 'transition state' that we define here as the half way contact; yet this point is on this level of consideration of minor importance. ${ }^{18}$ For moving 'up', the energy of the 'transition state' is equal to $E_{T}=4 \Delta / 2=2 \Delta$ and for moving 'down', $E_{T}=2 \Delta / 2=\Delta$. The difference between initial state energy and 'transition state' energy $\left(E_{G}-E_{T}\right)$ yields the activation barrier $A$, which then determines, within the Metropolis algorithm, the jump probabilities. In the example considered in (Fig. 5) we have $P_{\text {up }}=1 / 2 \exp [-\beta(3 \Delta-2 \Delta)], P_{\text {down }}=1 / 2$ $\exp [-\beta(3 \Delta-\Delta)]$ and $P_{\text {stay }}=1-1 / 2 \exp [-\beta \Delta]-1 / 2$ $\exp [-2 \beta \Delta]$, since $P_{\text {up }}+P_{\text {down }}+P_{\text {stay }}=1$. As usual, $\beta$ is the inverse temperature. In one Monte Carlo step, we scan the whole cluster according to the number of all particles which create this cluster.

The second column in Fig. 4a shows the fractal networks formed by conventional two-dimensional cluster-cluster aggregation, for different particle concentrations. While for $\varphi=0.01$ and 0.02 we are well below the percolation threshold, we are already above it for $\varphi=0.03$. Well below the threshold, the values of the fractal dimensions of the non-spanning network are between 1.6 and 1.7, being in agreement with the literature. At $\varphi_{c}$, due to the large finite size corrections, we obtained values between 1.8 and 1.9. If we allow for a smaller hit-and-stick probability of only $10 \%$, the results are qualitatively the same, but more dense networks resulting in a larger fractal dimension appear.

The third column of Fig. 4a shows the striking effect of coarsening on the conductivity. Already a slight rearrangement of the network in order to minimize surface energy, leads to pathway interruption and loss of percolation. This loss is augmented by subsequent sedimentation. Fig. $4 \mathrm{~b}$ shows the time evolution of the aggregate due to the coarsening process. The figure shows that the coarsening slows down quite rapidly, such that above 1000 Monte Carlo steps the structure remains unchanged.

Fig. 5 also shows the comparison of the one-sided contact with a two-sided contact. According to the above, for particles with two-sided contacts, the jump probability is quadratically reduced. This severe effect of packing on the coarsening is exactly what is observed: networks are much more stable with respect to coarsening at high $\varphi /$ small $R$. Fig. 4a gives examples of network development for less and more heavily packed situations; while for low $\varphi$ a severe coarsening occurs, coarsening is less significant for high $\varphi$.

It is experimentally observed (Fig. 3) that with higher volume fraction at constant $R$ or with smaller size at constant $\varphi$ the conductivity variations $(\delta \sigma)$ observed are much smaller, indeed, while the time constants do not change very much.

This leads to the following conclusions: We experimentally only observe those rearrangements that affect particles with one or two neighbors, while rearrangement effects involving particles with more neighbors are out of the experimental window. If clusters are already packed right from the beginning, the coarsening effect is only weak ( $\delta \sigma$ small) compared with 
the lowly packed situation. This is corroborated by Fig. 4 showing that in the first 1000 time steps the major rearrangement effects occur. Then even for the next 10000 steps the morphology does not really change.

The fact that we considered aggregation and coarsening to happen at very different time scales is justified by the fact that the first process should be determined by the particle diffusion coefficient in the liquid and the latter one by the surface diffusion coefficients of the particles. Experimental evidence for the latter is provided by the fact that the time constant of coarsening is (but not the conductivity variation) the same for PEG-150 and PEG-350 at given $\mathrm{SiO}_{2}$ volume fraction $\varphi$ (Fig. 2b).

What Fig. 4 also shows is the caging effect: obviously conductive liquid can be trapped within clusters and hence electrolyte contribution is lost in that way. This is one reason for the low conductivity at $\varphi>\varphi_{c}$ in Fig. 2. Another reason is the limited salt amount which leads to inactive particles blocking conducting pathways as well as to deactivation of salt for the transport in the liquid through adsorption by insulated particles. That this strongly depends on the network morphology is obvious by comparing the two curves in Fig. 1. The extremely abrupt and steep decrease of $\sigma_{m}$ corresponding to an extremely sharp peak in $\sigma_{m}(\varphi)$ often observed is probably caused by chain segmental motion and network cracking that can lead to extreme non-linear effects which are not included in our simplified treatment.

The Monte Carlo treatment confirms that already at low $\mathrm{SiO}_{2}$ volume fraction, conductive situations appear for the "soggy sand" electrolytes. Besides fast formation of percolating networks, network stability is a critical point. The network stability that is needed for the conductivity to become stationary requires highly packed percolation networks and hence high volume fractions of fine particles. An alternative not tackled here, is to make use of electrolyte/particle interactions (lyophilic networks) that would stabilize the network yet partially at the cost of local conductivity effects. It is believed that these modeling studies are also helpful for colloid phenomena in other contexts.

\section{Acknowledgements}

We have benefited from discussions with Dr Kosmas Kosmodis.

\section{References}

1 A. J. Bhattacharyya and J. Maier, Adv. Mater., 2004, 16, 811-814.

2 B. Kumar and S. J. Rodrigues, Solid State Ionics, 2004, 167, 91-97.

3 A. Bunde, W. Dieterich and H. E. Roman, Solid State Ionics, 1986, 18-19, 147-150.

4 A. J. Bhattacharyya, J. Maier, R. Bock and F. F. Lange, Solid State Ionics, 2006, 177, 2565-2568.

5 A. Jarosik, S. Hore, N. Kaskhedikar and J. Maier, Electrochim. Acta, 2010, submitted.

6 A. Jarosik, PhD thesis, University of Stuttgart, Germany, 2009.

7 The annealing removes most of the OH-groups of the so-called fumed silica $(7 \mathrm{~nm})^{6}$.

8 S. K. Das and A. J. Bhattacharyya, J. Phys. Chem. B, 2010, 114, 6830-6835.

9 S. R. Raghavan, M. W. Riley, P. S. Fedkiw and S. A. Khan, Chem. Mater., 1998, 10, 244-251.

10 J. Maier, Prog. Solid State Chem., 1995, 23, 171-263.

11 We do not consider the kinetics of the initial network formation as it is very fast in these examples.

12 J. Maier, J. Electrochem. Soc., 1987, 134, 1524-1535.

13 W. C. K. Poon and M. D. Haw, Adv. Colloid Interface Sci., 1997, 73, 71-126.

14 M. Kolb, R. Botet and R. Jullien, Phys. Rev. Lett., 1983, 51, 1123-1126; M. Kolb, Phys. Rev. Lett., 1984, 53, 1653-1656.

15 P. Meakin, Phys. Rev. Lett., 1983, 51, 1119-1122.

16 Fractals and Disordered Systems, ed. A. Bunde and S. Havlin, Springer-Verlag, Berlin, 1996.

17 The fact that we estimate the corner connection and the half-plane connection with the same energy contribution is very rough but is qualitatively justified if one considers particles to be spherical and the chain segments to be less stiff.

18 The artificial counting rules make sure that the intermediate state has an energy that is higher than or equal to the energies of the initial or final states. 